\title{
Broken Mean Stationarity and the Validity of the Dickey-Fuller Test: The Case of Controlled Inflation*
}

\author{
Daniel Ventosa-Santaulària** \\ Manuel Gómez-Zaldívar ${ }^{* * *}$
}

\begin{abstract}
This article proves the asymptotic efficiency of the Dickey Fuller (DF) test when the data generating process of the variable under consideration is in fact mean stationary with breaks. Monte Carlo simulations show that asymptotic properties remain valid for sample sizes of practical interest. Our results complement those already available in the literature, which can be summarized as follows: DF-type tests (i) over-accept the null hypothesis of unit root when there is a break in the trend-stationary process, and; (ii) over-reject the null hypothesis when there is a level/trend break in the unit root process. We illustrate the performance of the DF-t test by studying inflation rate series, a variable that should be stationary if the monetary authority follows an effective monetary policy: shocks are short-lived, therefore, inflation fluctuates randomly around specific goals.
\end{abstract}

Keywords: Dickey-Fuller Test, Mean Stationary Process, Structural Breaks.

JEL Codes: C12, C22, E31.

\footnotetext{
${ }^{*}$ Submitted in March 2008. Revised in May 2009.

** Corresponding author: Departamento de Economía y Finanzas, Universidad de Guanajuato. DCEA-Campus Marfil Fracc. I, El Establo, Guanajuato, Gto., C.P. 36250, Mexico. Phone and Fax (+52) (473) 735-2925. E-mail: daniel@ventosa-santaularia.com

*** Departamento de Economía y Finanzas, Universidad de Guanajuato.
} 


\section{Introduction}

Whether or not inflation follows a stationary process is an important and conflicting issue for a broad range of economic analyses and policymaking questions. For instance, evidence that inflation behaves as a stationary process may imply that it is being controlled by a certain monetary policy - the monetary authority is able to offset shocks that might induce significant deviations from a pre-specified inflation target. Moreover, if inflation rates are $I(1)$, then price levels would be $I(2)$. This in turn implies - for the long-run PPP relationship to hold - that either price levels and nominal exchange rate are cointegrated of order $(2,2)$ denoted $C I(2,2)$, or price levels are $C I(2,1)$ and that this linear combination is cointegrated with the nominal exchange rate.

The literature dealing with the statistical properties of inflation rate series is vast, and these have been analyzed from different perspectives. Gregoriou and Kontonikas (2006) actually assert that the inflation process in economies where the Central Bank adopted an explicit inflation targeting monetary policy should be stationary around the target, and find evidence to support their claim by running a unit root (UR) test that allows for a nonlinear mean reverting process in the alternative. Lai (1997) uses a modified DF test based on weighted symmetric least squares estimation to show that the use of different data frequencies and different price indexes to measure inflation can lead to conflicting evidence; his empirical results show considerable evidence of stationarity for monthly inflation rates, and mixed evidence for quarterly ones. Along the same lines, Culver and Papell (1997) and Basher and Westerlund (2006) accept the null hypothesis of stationarity by using a panel data UR test. In contrast, Bai and $\mathrm{Ng}$ (2004) focus on the common trend component of inflation rather than on the series themselves, and find mixed evidence with a new testing methodology, known as PANIC. A further possibility, as considered by Hassler and Wolters (1995) and Arize et al. (2005), is that inflation follows long-memory processes; this would explain why standard tests fail to reject the UR hypothesis. Persistence shifts in inflation have also been studied and there appears to be a change of regime in the series from an I(1) process to a mean stationary one (Sollis, 2001, Taylor, 2005, Chiquiar et al., 2006). Nevertheless, these results should be regarded with caution; Cavaliere and Taylor (2006) identified severe size distortions in persistence tests in the presence of a volatility shift.

The considerable effort devoted to discriminating between UR and stationarity has led to the current plethora of UR tests, yet, as Phillips and Xiao (1998) have pointed out: "The immense literature and diversity of UR tests can at times be confusing even to the specialist and presents a truly daunting prospect to the uninitiated. In consequence, much empirical work makes use of the simplest testing procedures because it is unclear from the literature [...] which tests, if any, are superior..." 
Hence, the standard DF remains the most popular methodology when testing for stationarity. Nevertheless, DF test is subject to certain amount of criticism which, in turn, has led theorists and practitioners to be skeptical about the conclusions to be drawn from it.

The contribution of this article is that of demonstrating that the standard DF test is asymptotically effective when used to differentiate between an $I(1)$ and a mean stationary process with breaks; both are relevant DGPs when studying inflation. ${ }^{1}$ We show that the asymptotic results also apply in finite samples by means of a Monte Carlo study. Furthermore, it is shown that the effects of location and break size and autocorrelation on the DF test, although not negligible in relatively small samples, could be corrected when a sample of at least 250 observations is used. Our theoretical results complement a vast literature concerning the effects of structural breaks in DF-type tests:

- Perron (1989) showed that the effectiveness of the UR tests decreases significantly in the presence of structural breaks. That is, if the true data generating process (DGP) of economic time series is in fact broken-trend stationary, UR tests would under-reject the null hypothesis. Montañés and Reyes (1998, 1999) and Leybourne and Newbold (2000) analyzed the asymptotic properties of DF-type tests when the process under analysis has a break in the trend function; they also found that the DF test is biased towards the non-rejection of the UR hypothesis for small sample sizes. Sen $(2001,2003)$ completed their results by studying the DF- $\mathcal{F}$ test (Dickey and Fuller, 1981) using the same DGP. In Kim et al.'s (2004b) study of the DF auxiliary regression with constant and trend using a broken trend stationary DGP, the authors observed that the nature and location of the break significantly affects the DF test. Montañés and Sansó (2001) studied the performance of the DF test using the same trend stationary process together with the inclusion of a seasonal pattern using dummy variables; they showed that, in spite of the seasonal component, the DF tends to reject the UR null hypothesis.

- Leybourne et al. (1998) proved that the DF-t test spuriously rejects the null hypothesis when the process not only includes a unit root but also a break, whether the latter is a level or a trend break. Sen (2008) extended Leybourne et al.'s (1998) results by proving that, when the process is a unit root with level or trend breaks, other DF statistics $(T(\hat{\rho}-1)$ and $\mathcal{F}$ test $)$ also spuriously rejects the null hypothesis.

- Hamori and Tokihisa (1997) reported that the DF test (with no constant and trend; see equation 2) spuriously over-rejects the null of unit root in the

\footnotetext{
${ }^{1}$ Under the null, inflation contains a UR; this implies an uncontrolled inflation process. Under the alternative, inflation follows a mean stationary process with level breaks; this implies that monetary policy actions ensure that inflation oscillates randomly around specific inflation targets - although the target may change throughout time.
} 
presence of variance shifts. Kim et al. (2002) proved that the same occurs with other specifications of the DF test (see equations 3 and 4 ). Kim et al. (2002), Busetti and Taylor (2003) and Sen (2009) proposed specific methodologies to control for these shifts in order to maintain adequate properties of the unit root tests.

Empirically, we contribute to reconciling disparate evidence on stationarity in inflation for OECD countries.

The article is organized as follows: in Section 2, we show the asymptotic behavior of the DF-t test as well as several particularly revealing properties of the asymptotic expressions. Section 3 presents Monte Carlo simulations. Section 4 presents an empirical application of the DF test using inflation rate series for the OECD countries, whilst conclusions are drawn in Section 5. Mathematical derivations are provided in the Appendix.

\section{The DF-t Test in the Presence of Structural Breaks}

DF-type tests consider (i) under the null hypothesis that the series contains a unit root, and; (ii) under the alternative hypothesis that the series is stationary; the process may be stationary around a constant (mean stationary) or a linear trend (trend stationary). Therefore, the DF auxiliary regression may include a constant and a deterministic trend as regressors (see equations 3 and 4). A relevant alternative hypothesis is therefore that of a stationary process that fluctuates around a constant that shifts abruptly: a mean stationary process with structural breaks. This process can be represented as follows:

$$
x_{t}=\mu_{x}+\sum_{i=1}^{N_{x}} \theta_{x} D U_{i, t}+u_{x t}
$$

where $\mu_{x}$ is a constant, $u_{x t}=\phi_{z} u_{x t-1}+\epsilon_{t},\left|\phi_{x}\right|<1, \epsilon_{t}$ is $i i d\left(0, \sigma_{\epsilon}^{2}\right)$, and $D U_{i, t}$ are dummy variables allowing for changes in the mean, that is, $D U_{i, t}=\mathbf{1}\left(t>T_{b_{i}}\right)$, where $\mathbf{1}(\cdot)$ is the indicator function, and $T_{b_{i}}$ is the unknown date of the $i^{\text {th }}$ break in $x$. We define the break fraction as $\lambda_{i}=\left(T_{b_{i}} / T\right) \in(0,1)$, where $T$ is the sample size. We use an $\operatorname{AR}(1)$ structure for the innovations $u_{x t}$ as in Kim et al. (2004a) and Noriega and Ventosa-Santaulària (2006), although it can also be assumed that innovations obey the (general-level) conditions stated in Phillips (1986). If we use the following four DF auxiliary regressions:

$$
\begin{aligned}
\Delta x_{t} & =\delta_{1} x_{t-1}+u_{t} \\
\Delta x_{t} & =\alpha+\delta_{2} x_{t-1}+u_{t} \\
\Delta x_{t} & =\alpha+\delta_{3} x_{t-1}+\beta t+u_{t} \\
\Delta x_{t} & =\alpha+\delta_{4} x_{t-1}+\beta t+\gamma \Delta x_{t-1}+u_{t},
\end{aligned}
$$

we can assert the following proposition: 
Proposition 1 Let $x_{t}$ be generated by DGP 1 and be used to estimate regression (2), (3), (4) or (5). Hence, the t-statistic associated with $\hat{\delta}_{i}$ diverges:

$$
t_{\hat{\delta_{i}}}=O_{p}\left(T^{\frac{1}{2}}\right) \quad \text { for } \quad i=1,2,3,4
$$

Proof See Appendix.

Proposition 1 states that the DF asymptotically maintains its power against the null hypothesis of unit root when the true DGP is a broken-mean stationary process.

Remark 1 Let $x_{t}$ be generated by DGP 1 , where only one break exists $\left(N_{x}=1\right)$ and occurs at the break date $T_{b}<T$, and be used to estimate regression (2). Hence, the t-statistic associated with $\hat{\delta}_{1}$ is:

$$
T^{-\frac{1}{2}} t_{\hat{\delta_{1}}} \stackrel{p}{\rightarrow}-\sqrt{\frac{\sigma_{u}^{2}\left(1-\rho_{1 x}\right)}{2\left[\mu_{x}^{2}+\theta_{x}\left(1-\lambda_{x}\right)\left(2 \mu_{x}+\theta_{x}\right)\right]+\sigma_{x}^{2}\left(1+\rho_{1 x}\right)}}
$$

where $\stackrel{p}{\rightarrow}$ indicates convergence in probability and $\rho_{1 x}$ is the first autocorrelation of $x_{t}$.

Figure 1(a) clearly illustrates the implication of the formula of the asymptotic t-statistic associated with $\hat{\delta}$ in equation (2): where the structural break is positive, the DF test decreases its power, the larger the size of the break and the smaller the break fraction. Furthermore, where the structural break is negative, the DF test decreases its power when there is an increase in the size of the break only when $\left(\mu_{x}<-\theta_{x}\right)$; analogously, an increase in the break fraction biases the DF test toward the acceptance of the null hypothesis if $\left(\frac{-2 \mu_{x}}{\theta_{x}}<1\right)$. Similarly, Figure 1(b) shows that positive autocorrelation decreases the power of the test, and negative autocorrelation biases the DF test toward the rejection of the null hypothesis.

Our results complement those of Montañés and Reyes $(1998,1999)$, Leybourne and Newbold (2000) and Sen $(2001,2003)$ (where it is proved that the DF-type tests spuriously over-accept the null hypothesis of unit root whenever the variable is generated as a trend stationary process with breaks) as well as those of Leybourne et al. (1998) and Sen (2008) (where it is proved that the DF-type tests spuriously over-reject the null whenever the variable is generated as a unit root process with a break).

The Monte Carlo section will show that the problems such as an increase in autocorrelation, the size and location of the breaks can be overcome by increasing the sample size. 
Figure 1

T-statistic behavior according to the size and location of the break, and the degree of autocorrelation in the noise

(a)

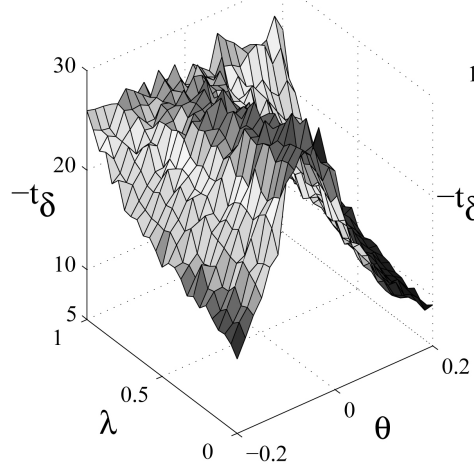

(b)

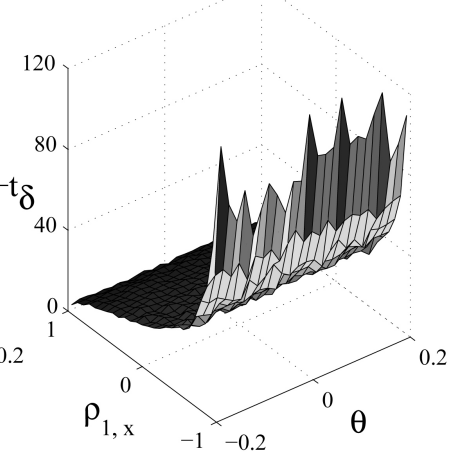

\section{A Monte Carlo Study}

We present a Monte Carlo study in which the finite-sample behavior of the DF test is analyzed in the case where the variable under examination is mean stationary with breaks - see equation (1). Table 1 shows the rejection rates of the null hypothesis of the DF test with neither constant nor time trend, see equation (2). The computed parameter values selected were similar to those obtained in empirical estimates. ${ }^{2}$ Sample sizes range from $T=25$ to $T=500$, whilst the number of replications is $R=10,000$. The rejection rates presented in this table are consistent with the theoretical results obtained in the previous section: firstly, columns 2 and 3 exemplify the effect of autocorrelation. The only difference between the DGPs in these two columns is that the first uses i.i.d innovations whereas the second uses a stationary $A R(1)$ process. It is clear that rejection rates decrease in the presence of positive autocorrelation. Secondly, columns 5 and 6 illustrate that with a smaller positive break, rejection rates are greater, at least in the case of small sample sizes. Thirdly, columns 7 and 8 show the location of the break effect. With a positive break, the smaller the break fraction, the smaller the rejection rates. Finally, columns 3 and 4 illustrate the effect of adding lags in the auxiliary regression - $D G P_{2}^{a}$ is identical to $D G P_{2}$; instead of using the test regression (2), we use an augmented version of it, that is: $\Delta x_{t}=\delta x_{t-1}+\gamma \Delta x_{t-1}+u_{t}$. Results

\footnotetext{
${ }^{2}$ The parameters of the different DGPs are shown in Appendix B.
} 
indicate that the inclusion of lags reduces the power of the test - we performed such comparison for several $A R(1)$ models where $\rho$ varies from -0.95 to 0.95 ; the estimation results were qualitatively the same as those reported in Table 1 . These results are in line with DeJong et al. (1992). Furthermore, Harris (1992) showed that increasing the number of lags leads to an ADF test with size close to its nominal value. Therefore, the use of lags corrects the level of the test but lessens its power. The Monte Carlo experiment shows that all the power distortions caused by the break size, break location and autocorrelation dissipate once the sample size reaches 250 observations.

Table 1

Monte Carlo experiment: rejection rates of the t-ratio statistic when the variable follows a broken-mean stationary process

\begin{tabular}{c|c|c|c|c|c|c|c}
\hline $\mathrm{T}$ & $D G P_{1}$ & $D G P_{2}$ & $D G P_{2}^{a}$ & $D G P_{3}$ & $D G P_{4}$ & $D G P_{5}$ & $D G P_{6}$ \\
\hline 25 & 0.83 & 0.03 & 0.03 & 0.26 & 0.00 & 0.17 & 0.92 \\
\hline 50 & 0.99 & 0.07 & 0.07 & 0.93 & 0.00 & 0.88 & 0.99 \\
\hline 100 & 1.00 & 0.26 & 0.24 & 1.00 & 0.18 & 1.00 & 1.00 \\
\hline 250 & 1.00 & 0.97 & 0.94 & 1.00 & 1.00 & 1.00 & 1.00 \\
\hline 500 & 1.00 & 1.00 & 1.00 & 1.00 & 1.00 & 1.00 & 1.00 \\
\hline
\end{tabular}

We computed a second Monte Carlo exercise (the DGPs are the same as those of the first experiment) for another DF test, the DF- $\mathcal{F}$ see (see Dickey and Fuller, 1981). The auxiliary regression equation and the relevant null hypothesis are, $y_{t-1}=\alpha+\delta y_{t-1}+\epsilon_{y t}$ and $\mathcal{H}_{0}: \alpha=0, \delta=1$, respectively. Table 2 shows that the rejection rates are similar to those obtained in the previous simulation, where the DF-t statistic was used. It is noteworthy that, when there is autocorrelation and the auxiliary regression controls it (see column 4 ) the DF- $\mathcal{F}$ test preserves its power even in small samples, contrary to the DF-t statistic.

This can be seen as evidence that DF-type tests are robust to the presence of shifts in the constant of a mean stationary process.

Table 2

Monte Carlo experiment: rejection rates of the DF- $\mathcal{F}$ statistic when the variable follows a broken-mean stationary process.

\begin{tabular}{c|c|c|c|c|c|c|c}
\hline $\mathrm{T}$ & $D G P_{1}$ & $D G P_{2}$ & $D G P_{2}^{a}$ & $D G P_{3}$ & $D G P_{4}$ & $D G P_{5}$ & $D G P_{6}$ \\
\hline 25 & 0.78 & 0.01 & 0.91 & 0.37 & 0.00 & 0.83 & 0.79 \\
\hline 50 & 0.99 & 0.03 & 0.99 & 0.99 & 0.04 & 1.00 & 0.99 \\
\hline 100 & 1.00 & 0.17 & 1.00 & 1.00 & 0.96 & 1.00 & 1.00 \\
\hline 250 & 1.00 & 0.90 & 1.00 & 1.00 & 1.00 & 1.00 & 1.00 \\
\hline 500 & 1.00 & 1.00 & 1.00 & 1.00 & 1.00 & 1.00 & 1.00 \\
\hline
\end{tabular}




\section{Empirical Evidence}

This section shows the theoretical results discussed earlier by way of an empirical application; we analyze the results of the DF-t test applied to inflation rate series corresponding to OECD countries. Quarterly inflation rate series ${ }^{3}$ were constructed from monthly Consumer Price Index data retrieved from the International Monetary Fund's International Financial Statistics data; the sample period for these countries being 1957:01 to 2005:12.

Firstly, Figure 2 shows the quarterly inflation rate series of a subsample of the countries and their abrupt structural changes in the mean of the series estimated using the methodology proposed by Bai and Perron $(1998,2003) .{ }^{4}$ This figure is shown to exemplify that broken mean stationarity is a suitable DGP for an inflation series. Average inflation rates were higher during the 1970s (around the oil price shock), then they decreased steadily during the 1980s and went further down during the 1990s and the 2000s.

\footnotetext{
${ }^{3}$ Quarterly inflation series were computed as the logged differences of CPI at three-monthly intervals.

${ }^{4}$ This methodology is valid only when the series under analysis is $I(0)$.
} 
Figure 2

Inflation as a broken mean stationary process; Inflation (solid line), time-varying mean (dotted line)
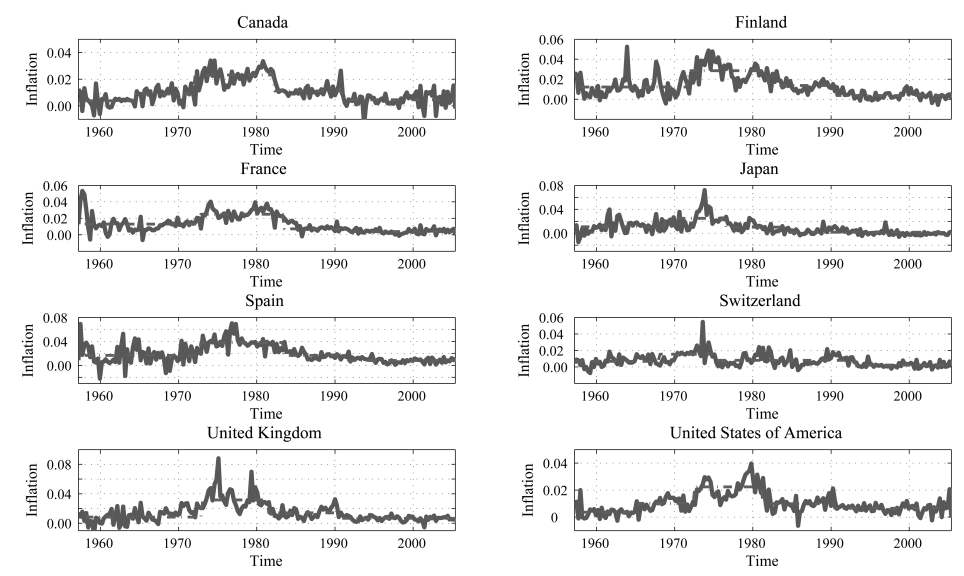

Secondly, Table 3 presents the results of the DF-t test - without a constant or time trend. ${ }^{5}$ The results are in line with the asymptotic and finite value properties of the DF-t test. On the one hand, the first three columns of this table illustrate the effect of the sample size. For all the countries presented in this table, evidence in favor of broken mean stationarity is stronger the larger the sample size. Nevertheless, the associated Q-statistics indicate that residuals are not white noise. On the other hand, the last column shows the results of the test when correcting for autocorrelation. In this case, the DF-t test without intercept or trend includes the number of lags selected by the Schwarz information criterion (SIC). The empirical application reflects the properties described in the asymptotic and finite sample section; the test suffers from loss of power when lags are included. Evidence in favor of broken mean stationarity is found in only 10 out of the 17 countries presented. The Q-statistics indicate that most of the residual can now be considered as white noise.

\footnotetext{
${ }^{5}$ The test was also performed under different specifications (DF with intercept, as well as intercept and time trend), providing similar results. These results are available upon request.
} 


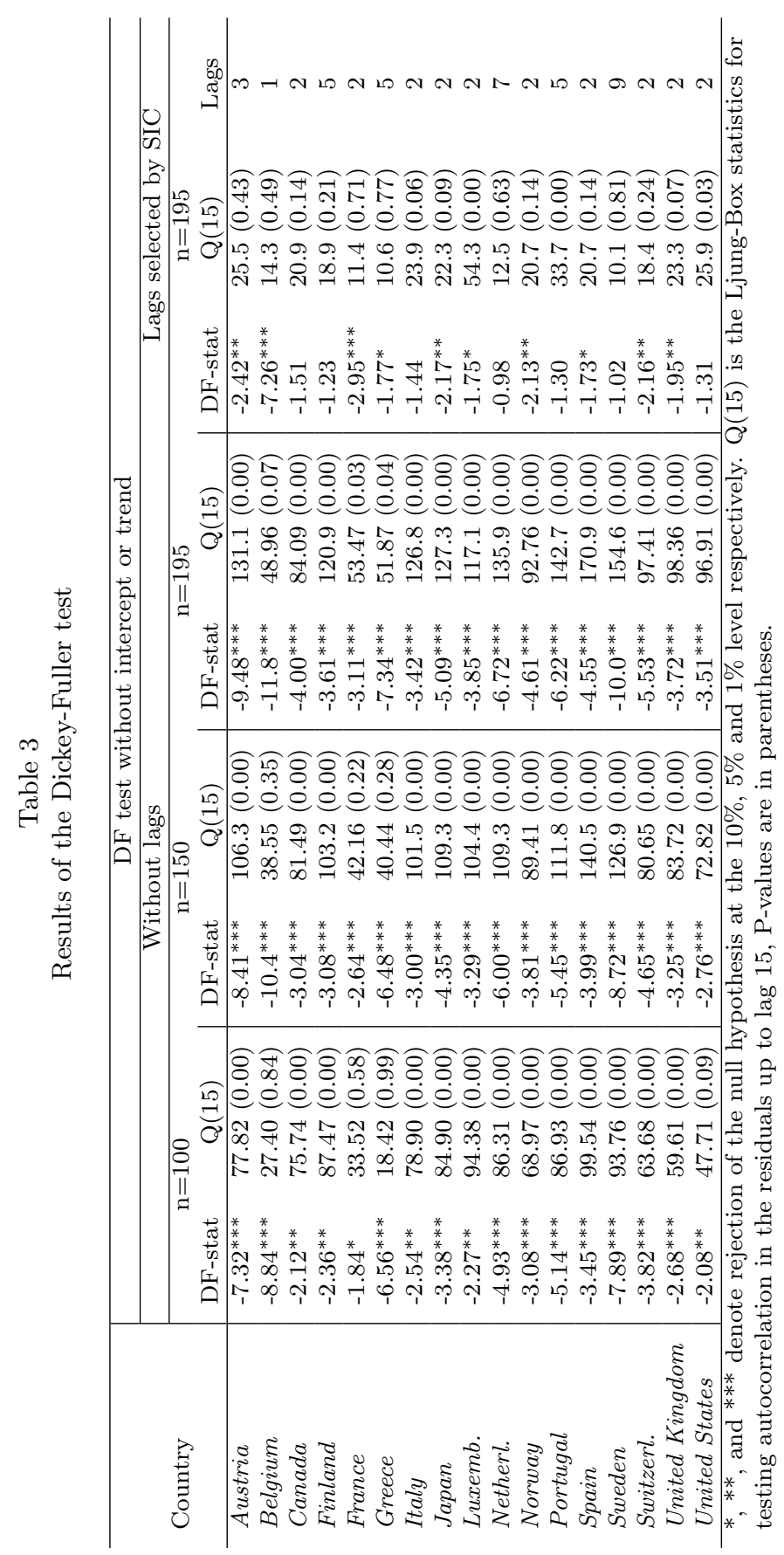




\section{Conclusions}

Structural breaks do affect the performance of UR tests. Nevertheless, we have shown the robustness of the DF test in the presence of structural breaks where the DGP of the series under analysis is mean stationary with breaks. In that case, the t-statistic of the DF test diverges at rate $\sqrt{T}$ implying that the null will, at some point, be rejected.

Our results complement those available in the literature. It is now well-known that DF-type tests over-accept the null hypothesis of unit root when the process is in fact broken-trend stationary (see Perron, 1989, Montañés and Reyes, 1998, 1999, Leybourne and Newbold, 2000, Sen, 2001, 2003). The converse problem, i.e. over-rejection of the null hypothesis of the DF-type tests when the process is a unit root with level or trend breaks, has also been well documented (see Leybourne et al., 1998, Sen, 2008). All these results show that the DF-type test can provide misleading evidence in the presence of level breaks, whether the breaks are under the null or under the alternative hypothesis. Our results are in sharp contrast with this; we prove that, asymptotically, the DF t-statistic maintains its power when the variable is generated as a broken-mean stationary process; by means of a Monte Carlo experiment we show that the asymptotics of the DF-t statistic approximate fairly well the finite-sample behavior of the test for sample sizes of practical interest.

However, our asymptotic results also prove that the DF test is sensitive not only to the size of breaks, but also to their sign and their location, as well as to autocorrelation. This is in line with the results in the literature (see, for example, DeJong et al., 1992).

Monte Carlo experiments and empirical evidence show that the DF-t test provides adequate results when the sample size is sufficiently large. In fact, the DF is more sensitive to the presence of autocorrelation, regardless of the type of the break, although this phenomenon can also be alleviated by increasing the size of the sample or simply by running an augmented DF test. UR testing can be reliably performed using a simple DF test if the sample size is large enough. In the specific case of inflation rates, the alternative of broken mean stationarity may be understood as the existence of an inflation targeting regime with time-varying inflation targets. Evidence of this is found in OECD countries.

\section{References}

Arize, A., Malindretos, J., \& Nam, K. (2005). Inflation and structural change in 50 developing countries. Atlantic Economic Journal, 33(4):461-471.

Bai, J. \& Ng, S. (2004). A PANIC attack on unit roots and cointegration. Econometrica, 72(4):1127-1177. 
Bai, J. \& Perron, P. (1998). Estimating and testing linear models with multiple structural changes. Econometrica, 66(1):47-78.

Bai, J. \& Perron, P. (2003). Computation and analysis of multiple structural change models. Journal of Applied Econometrics, 18(1):1-22.

Basher, S. A. \& Westerlund, J. (2006). Is there really a unit root in the inflation rate? More evidence from panel data models. MPRA paper 136.

Busetti, F. \& Taylor, A. (2003). Variance shifts, structural breaks, and stationarity tests. Journal of Business and Economic Statistics, 21(4):510-531.

Cavaliere, G. \& Taylor, R. (2006). Testing for a change in persistence in the presence of a volatility shift. Oxford Bulletin of Economics and Statistics, 68(s1):761-781.

Chiquiar, D., Noriega, A., \& Ramos-Francia, M. (2006). A time series approach to testing for a change in inflation persistence: The Mexican experience. Mimeo, Banco de México; forthcoming at Applied Economics.

Culver, S. \& Papell, D. (1997). Is there a unit root in the inflation rate? Evidence from sequential break and panel data models. Journal of Applied Econometrics, 12(4):435-444.

DeJong, D., Nankervis, J., Savin, N., \& Whiteman, C. (1992). The power problems of unit root tests in time series with autoregressive errors. Journal of Econometrics, 53(1-3):323-344.

Dickey, D. \& Fuller, W. (1979). Distribution of the estimators for autoregressive time series with a unit root. Journal of the American Statistical Association, $74: 427-31$

Dickey, D. \& Fuller, W. (1981). Likelihood ratio statistics for autoregressive time series with a unit root. Econometrica, 49:1057-1072.

Gregoriou, A. \& Kontonikas, A. (2006). Inflation targeting and the stationarity of inflation: New results from an ESTAR unit root test. Bulletin of Economic Research, 58(4):309-322.

Hamori, S. \& Tokihisa, A. (1997). Testing for a unit root in the presence of a variance shift. Economics Letters, 57(3):245-253.

Harris, R. (1992). Testing for unit roots using the augmented Dickey-Fuller test: Some issues relating to the size, power and the lag structure of the test. Economics Letters, 38(4):381-386. 
Hassler, U. \& Wolters, J. (1995). Long memory in inflation rates: International evidence. Journal of Business \& Economic Statistics, 13(1):37-45.

Kim, T., Lee, S., \& Newbold, P. (2004a). Spurious regressions with stationary processes around linear trends. Economics Letters, 83:257-262.

Kim, T., Leybourne, S., \& Newbold, P. (2002). Unit root tests with a break in innovation variance. Journal of Econometrics, 109(2):365-387.

Kim, T., Leybourne, S., \& Newbold, P. (2004b). Behaviour of Dickey-Fuller unit-root tests under trend misspecification. Journal of Time Series Analysis, $25(5): 755-764$.

Lai, K. (1997). On the disparate evidence on trend stationarity in inflation rates: A reappraisal. Applied Economics Letters, 4(5):305-309.

Leybourne, S., C. Mills, T., \& Newbold, P. (1998). Spurious rejections by DickeyFuller tests in the presence of a break under the null. Journal of Econometrics, 87(1):191-203.

Leybourne, S. \& Newbold, P. (2000). Behavior of Dickey-Fuller t-tests when there is a break under the alternative hypothesis. Econometric Theory, 16(05):779789 .

Montañés, A. \& Reyes, M. (1998). Effect of a shift in the trend function on Dickey-Fuller unit root tests. Econometric Theory, 14:355-363.

Montañés, A. \& Reyes, M. (1999). The asymptotic behaviour of the Dickey-Fuller tests under the crash hypothesis. Statistics and Probability Letters, 42(1):81-89.

Montañés, A. \& Sansó, A. (2001). The Dickey-Fuller test family and changes in the seasonal pattern. Annales d'Économie et de Statistique, 61:73-90.

Noriega, A. \& Ventosa-Santaulària, D. (2006). Spurious regression under broken trend stationarity. Journal of Time Series Analysis, 27:671-684.

Perron, P. (1989). The great crash, the oil price shock and the unit root hypothesis. Econometrica, 57:1361-1401.

Phillips, P. (1986). Understanding spurious regressions in econometrics. Journal of Econometrics, 33:311-340.

Phillips, P. \& Xiao, Z. (1998). A primer on unit root testing. Journal of Economic Surveys, 12(5):423-470.

Sen, A. (2001). Behaviour of Dickey-Fuller F-tests under the trend-break stationary alternative. Statistics and Probability Letters, 55(3):257-268. 
Sen, A. (2003). On unit-root tests when the alternative is a trend-break stationary process. Journal of Business and Economic Statistics, 21(1):174-184.

Sen, A. (2008). Behaviour of Dickey-Fuller tests when there is a break under the unit root null hypothesis. Statistics and Probability Letters, 78(6):622-628.

Sen, A. (2009). Unit root tests in the presence of an innovation variance break that has power against the mean break stationary alternative. Statistics and Probability Letters, 79(3):354-360.

Sollis, R. (2001). US and UK inflation: Evidence on structural change in the order of integration. Working Paper, Department of Economics and Ireland Trinity College Dublin.

Taylor, R. (2005). Fluctuation tests for a change in persistence. Oxford Bulletin of Economics and Statistics, 67(2):207-230. 


\section{Appendix A: Proof of Proposition 1}

We present a guide on how to obtain the order in probability of one of the four t-statistics appearing in Proposition (1), by using the DF regression (2) for which $\Delta x_{t}=\sum_{i=1}^{N_{x}} \theta_{i} D P_{i t}+u_{x t}-u_{x t-1}$ where $D P_{i, t}=D U_{i, t}-D U_{i, t-1}$. The other three cases follow the same steps. Proof as such was provided with the aid of Mathematica 4.1 software. $^{6}$

We shall now describe the process involved in establishing the aforementioned proof. The DF regression equation shall be $\Delta x_{t}=\alpha+\delta x_{t-1}+u_{t}$ in matrix form: $\Delta X=X_{1} \beta+u$.

The vector of $O L S$ estimators is $\widehat{\beta}=(\widehat{\alpha} \widehat{\delta})^{\prime}=\left(X_{1}^{\prime} X_{1}\right)^{-1} X_{1}^{\prime} \Delta X$, and the $t$ statistic of interest $t_{\widehat{\delta}}=\widehat{\delta}\left[\widehat{\sigma}_{u}^{2}\left(X_{1}^{\prime} X_{1}\right)_{22}^{-1}\right]^{-1 / 2}$, where $\left(X_{1}^{\prime} X_{1}\right)_{22}^{-1}$ is the $2^{\text {nd }}$ diagonal element of $\left(X_{1}^{\prime} X_{1}\right)^{-1}$ and $\widehat{\sigma}_{u}^{2}=T^{-1} \sum_{t=1}^{T} \widehat{u}_{t}^{2}=T^{-1} \sum_{t=1}^{T}\left(\Delta x_{t}-\widehat{\alpha}-\widehat{\delta} x_{t-1}\right)^{2} \cdot t_{\widehat{\delta}}$ is a function of the following expressions:

\begin{tabular}{|l|l|}
\hline$\sum_{t=1}^{T} \Delta x_{t}=O_{p}(1)$ & $\sum_{t=1}^{T}\left(\Delta x_{t}\right)^{2}=O_{p}(T)$ \\
\hline$\sum_{t=1}^{T} x_{t-1}=O_{p}(T)$ & $\sum_{t=1}^{T} x_{t}^{2}=O_{p}(T)$ \\
\hline$\sum_{t=1}^{T} \Delta x_{t} x_{t-1}=O_{p}(T)$ & $\sum_{t=1}^{T} x_{t-1} \cdot t=O_{p}\left(T^{2}\right)$ \\
\hline$\sum_{t=1}^{T} \Delta x_{t} \cdot t=O_{p}(T)$ & $\sum_{t=1}^{T} \Delta x_{t} \Delta x_{t-1}=O_{p}(T)$ \\
\hline
\end{tabular}

We can fill the matrix $\left(X_{1}^{\prime} X_{1}\right)$ as well as the vector $\left(X_{1}^{\prime} Y\right)$ and then compute the OLS parameter estimates $\beta=\left(X_{1}^{\prime} X_{1}\right)^{-1} X_{1}^{\prime} \Delta X$ and the t-statistic associated with $\delta$. The program computes the asymptotics. ${ }^{7}$

\footnotetext{
${ }^{6}$ The corresponding codes are available at http://www. ventosa-santaularia.com/VSG.zip.

${ }^{7}$ The Mathematica codes yield exact asymptotic expressions.
} 


\section{Appendix B: DGPs of the Monte Carlo Experiment}

The values of the parameters in the DGPs are as follows:

1. All DGPs: $\sigma_{\epsilon}=0.02, \mu_{x}=0.05$;

2. $D G P_{1}: \lambda_{1}=0.25 \lambda_{2}=0.75 \theta_{1}=0.09 \theta_{2}=-0.09 \rho_{x}=0$;

3. DGP $P_{2}: \lambda_{1}=0.25 \lambda_{2}=0.75 \theta_{1}=0.09 \theta_{2}=-0.09 \rho_{x}=0.9$;

4. $D G P_{2}^{a}$ : identical to $D G P_{2}$. Rejection rates obtained from using a different auxiliary regression: $\Delta x_{t}=\delta x_{t-1}+\gamma \Delta x_{t-1}+u_{t}$;

5. $D G P_{3}: \lambda_{1}=0.50 \theta_{1}=0.195 \rho_{x}=0$;

6. DGP $P_{4}: \lambda_{1}=0.50 \theta_{1}=0.495 \rho_{x}=0$;

7. $D G P_{5}: \lambda_{1}=0.10 \theta_{1}=0.15 \rho_{x}=0$;

8. DGP $: \lambda_{1}=0.90 \theta_{1}=0.15 \rho_{x}=0$. 